\title{
Nullité du loyer et créance en remboursement du trop-versé
}

Labsence dusage de la formule officielle prévue à lart. 270 al. 2 CO entraîne la nullité du loyer. Le locataire peut alors agir en fixation judiciaire du loyer initial et en remboursement de léventuel trop-versé selon les règles sur lenrichissement illégitime. Cette contribution examine plusieurs questions controversées relatives à la prescription de la créance en remboursement du locataire.

Catégories d'articles: Contributions

Domaines juridiques : Contrat de bail et de bail à ferme 


\section{Table des matières}

I. Introduction

II. Le délai relatif de l'art. 67 al. $1 \mathrm{CO}$

III. Le délai absolu de l'art. 67 al. 1 CO

IV. Les paiements volontaires au sens de l'art. 63 al. $1 \mathrm{CO}$

V. L'augmentation du loyer après la conclusion du contrat

VI. L'intérêt à agir même lorsque la créance est prescrite

VII. Le droit transitoire en matière de prescription

VIII. Conclusion

\section{Introduction}

[1] Selon l'art. 270 al. 2 du Code des obligations (CO), en cas de pénurie de logements, les cantons peuvent rendre obligatoire l'usage de la formule officielle évoquée à l'art. $269 \mathrm{~d}$ pour la conclusion de tout nouveau bail ${ }^{1}$. L'absence d'usage de la formule officielle, l'usage d'une formule officielle qui ne contient pas toutes les informations nécessaires ou la notification de la formule officielle plus de trente jours après l'entrée du locataire dans les locaux entraînent la nullité du loyer (nullité partielle ; art. 20 al. $2 \mathrm{CO})^{2}$.

[2] La nullité peut être invoquée en tout temps, sous réserve d'abus de droit manifeste (art. 2 al. 2 du Code civil [CC] $)^{3}$. On est en présence d'un abus de droit manifeste par exemple "lorsque le preneur s'est rendu compte du vice de forme et s'est abstenu de protester dans le dessein d'en tirer, le cas échéant, ultérieurement profit » ou « lorsque le locataire a renoncé expressément et en toute connaissance de cause à la notification de la formule officielle et a exécuté de son plein gré l'accord conclu $»^{4}$. En revanche, le seul fait de s'être acquitté du loyer pendant une longue période ne constitue pas un abus de droit manifeste ${ }^{5}$.

[3] En cas de nullité du loyer, le locataire peut agir en fixation judiciaire du loyer initial et en restitution de l'éventuel trop-versé selon les règles sur l'enrichissement illégitime ${ }^{6}$. La créance en restitution du trop-versé se prescrit par trois ans à compter du jour où le locataire a eu connais-

1 Sur le champ d'application de cette disposition, voir David Lachat/Pierre Stastny, Le bail à loyer, Lausanne 2019, p. 489 s. N 3.1.1 ss; Pierre Tercier/Laurent Bieri/Blaise Carron, Les contrats spéciaux, 5 ème éd., Genève/ Zurich/Bâle 2016, p. 294 ss N 2171 ss. Sur la notion de pénurie de logements, voir Patricia Dietschy-Martenet, in : François Bohnet/Blaise Carron/Marino Montini (édit.), Commentaire Pratique - Droit du bail à loyer et à

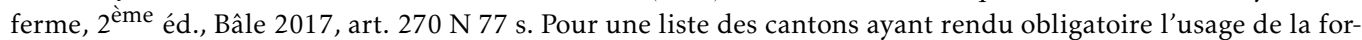
mule officielle, voir : https://www.bwo.admin.ch/bwo/fr/home/mietrecht/mietrecht--politik.html (consulté le 20.01.2020).

2 ATF 140 III 583 consid. 3.2.1 ; ATF 137 III 547 consid. 2.3 ; ATF 124 III 62 consid. 2a ; ATF 120 II 341 consid. 5; Dietschy-Martenet (note 1), art. $270 \mathrm{~N} 81$; Tercier/Bieri/Carron (note 1), p. 295 N 2173; Lachat/Stastny (note 1), pp. 493-494 N 3.4 ss ; Thomas Koller, Die mietrechtliche Rechtsprechung des Bundesgerichts im Jahr 2011, RJB 149/2013, p. 21 ss, p. 60 ; SÉBAstien FetTer, La contestation du loyer initial, Berne 2005, p. 101 ss N 219 ss.

3 Sur l'abus de droit à se prévaloir d'un vice de forme, voir ATF 140 III 583 consid. 3.2.4 ; ATF 138 III 401 consid. 2.4 ; ATF 137 III 547 consid. 2.3 ; arrêt du TF 4A_571/2017 du 10 juillet 2018 consid. 4.2 ; arrêt du TF 4A_517/2014 du 2 février 2015 consid. 4.1.3; Dietschy-Martenet (note 1), art. 270 N 81a. Le délai de 30 jours prévu à l'art. 270 al. 1 CO ne s'applique pas. Voir à cet égard arrêt du TF 4A_674/2012 du 23 septembre 2013 consid. 2; Dietschy-Martenet (note 1), art. $270 \mathrm{~N} 81 \mathrm{~b}$

4 Arrêt du TF 4A_254/2016 du 10 juillet 2017 consid. 4.1.3.

5 ATF 138 III 401 consid. 2.3.3.

6 ATF 140 III 583 consid. 3.2.3 ; Lachat/Stastny (note 1), p. 495 N 3.5.1; Tercier/Bieri/Carron (note 1), p. 295 N 2173. Sur la distinction entre les créances en remboursement de nature contractuelle et les créances fondées sur les règles de l'enrichissement illégitime, voir en particulier ATF 137 III 243 consid. 4 et ArIANe Morin, La restitution des paiements en matière de bail, $15^{\text {ème }}$ Séminaire sur le droit du bail, Neuchâtel 2008, p. 143 ss, p. 145 ss. 
sance de son droit de répétition, et dans tous les cas par dix ans dès la naissance de ce droit (art. 67 al. $1(\mathrm{CO})^{7}$. Il appartient au bailleur d'apporter la preuve que la prescription est acquise ${ }^{8}$.

[4] Cette contribution se penche d'abord sur le point de départ du délai relatif de trois ans, qui a déjà été discuté dans plusieurs arrêts du Tribunal fédéral, puis sur le point de départ du délai absolu de dix ans, qui a donné lieu à des décisions cantonales contradictoires. Elle traite ensuite de la portée de l'art. $63 \mathrm{CO}$, de l'effet d'une augmentation ultérieure du loyer, de l'intérêt du locataire à agir en fixation du loyer initial alors que la créance est prescrite, et du droit transitoire en matière de prescription.

\section{Le délai relatif de l'art. 67 al. $1 \mathrm{CO}$}

[5] Le délai de prescription de trois ans de l'art. 67 al. 1 CO commence à courir lorsque le locataire connaît suffisamment d'éléments pour fonder et motiver son action en justice, laquelle n'a pas besoin d'être chiffrée (art. $85 \mathrm{CPC}$ ) ${ }^{9}$. Le locataire doit avoir une connaissance effective de sa prétention et pas seulement avoir pu ou dû la connaître en faisant preuve de l'attention commandée par les circonstances ${ }^{10}$.

[6] Selon le Tribunal fédéral, la connaissance effective intervient lorsque le locataire « sait que l'absence de cette formule, respectivement de l'indication du loyer du locataire précédent ou de la motivation de la hausse, entraîne la nullité du loyer initial, que le loyer qu'il a versé était trop élevé et qu'il était, partant, abusif ${ }^{11}$. Il n'est en revanche pas nécessaire que le locataire connaisse précisément le loyer du précédent locataire ${ }^{12}$.

7 ATF 140 III 583 consid. 3.2.3 ; arrêt du TF 4A_254/2016 du 10 juillet 2017 consid. 3; arrêt du TF 4A_517/2014 du 2 février 2015 consid. 4.1.2 ; arrêt du TF du 18 octobre 2011, CdB 1/02 pp. 1-2, p. 2 ; Dietschy-Martenet (note 1), art. $270 \mathrm{~N}$ 81a; Lachat/Stastny (note 1), p. 498 N 3.6.1; Tercier/Bieri/Carron (note 1), p. 297 N 2186. En cas de nullité du loyer initial faute d'utilisation de la formule officielle, l'art. 270e CO ne semble pas applicable. Voir aussi Pierre Stastny, Jurisprudence choisie en matière de bail, Plaidoyer 2/2014, p. 36 ss, p. 39. Par conséquent, s'agissant des intérêts, il convient d'appliquer les principes valables en matière de remboursement de l'enrichissement illégitime. Sur ces principes, voir Françors BoHnet, Actions civiles, volume 2, 2 ème éd., Bâle 2019, § 3 N 42; Benoît Chappuis, in : Luc Thévenoz/Franz Werro (édit.), Commentaire romand - Code des obligations I, art. 1-529 CO, 2 ème éd., Bâle 2012, art. 64 N 5 ss ; Peter Gauch/Walter Schluep/Jörg Schmid, Schweizerisches Obligationenrecht Allgemeiner Teil, 10 ème éd., Zurich/Bâle/Genève 2014, p. 384 ss N 1525; Hermann Schulin, in : Heinrich Honsell/Nedim Peter Vogt/Wolfgang Wiegand (édit.), Basler Kommentar, Obligationenrecht I, art. 1-529 OR, 6 ème éd., Bâle 2015, art. 64 N 4b. La situation est différente en cas de contestation d'un loyer initial valablement notifié (art. 270 al. 1 CO). Dans ce cas, l'art. 270e CO s'applique et la créance du locataire porte intérêt (art. $104 \mathrm{CO}$ ) dès l'entrée en force du jugement. Voir Lachat/Stastny (note 1), p. 488 N 2.6.8, et les références citées. Si le bailleur est condamné, un nouveau délai de 10 ans commence depuis l'entrée en force du jugement en application de l'art. 137 al. 2 CO. Voir par exemple LaCHAt/Stastny (note 1), p. 498 N 3.6.2, note 216. À certaines conditions, le locataire peut invoquer une créance prescrite en compensation (art. 120 al. 3 CO).

ATF 140 III 583 consid. 3.2.3 ; Dietschy-Martenet (note 1), art. 270 N 81a.

9 ATF 140 III 583 consid. 3.2.3 et 3.3.1 ; arrêt du TF 4A_517/2014 du 2 février 2015 consid. 4.1.2. Dans l'ATF 137 III 547 consid. 2.3, puis dans l'arrêt du TF 4A_38/2013 du 12 avril 2013 consid. 2.1, le Tribunal fédéral avait affirmé que lorsque le locataire apprend le vice de forme, il doit « agir sans retard » ou « dans un délai raisonnable ». Dans l'ATF 140 III 583 consid. 3.3.1, le Tribunal fédéral a précisé (à raison) que ces affirmations « n'ont pas de portée dans le cadre de l'examen du respect du délai annuel [trois ans depuis le $1^{\mathrm{er}}$ janvier 2020] fixé par l'art. 67 al. 1 CO ». Dans ce sens également arrêt du TF 4A_254/2016 du 10 juillet 2017 consid. 4.1.2.

10 ATF 140 III 583 consid. 3.3.1 ; ATF 129 III 503 consid. 3.4 ; ATF 109 II 433 consid. 2 ; arrêt du TF 4A_254/2016 du 10 juillet 2017 consid. 3.1.3.1.

11 Arrêt du TF 4A_517/2014 du 2 février 2015 consid. 4.1.2. Voir également ATF 140 III 583 consid. 3.3.

12 Arrêt du TF 4A_517/2014 du 2 février 2015 consid. 4.2 ; Blaise Carron, Droit du Bail 2015, p. 36 ss, p. 37 ; Dietschy-Martenet (note 1), art. 270 N 81a. 
[7] Le fait que le locataire a consulté un mandataire professionnel auparavant dans le cadre d'un litige en droit du bail sans lien avec le montant du loyer initial ne permet pas de considérer que le vice de forme a été porté à sa connaissance à ce moment-là ${ }^{13}$.

\section{Le délai absolu de l'art. 67 al. 1 CO}

[8] Le point de départ du délai de prescription de dix ans depuis la naissance du droit (art. 67 al. 1 CO) fait l'objet d'une controverse, qui n'a apparemment pas encore été tranchée par le Tribunal fédéral.

[9] D'après la Chambre des baux et loyers de la Cour de justice du canton de Genève et une partie de la doctrine, la créance du locataire en remboursement du trop-versé doit être considérée comme une prétention unique qui se prescrit dans un délai de dix ans depuis la conclusion du contrat $^{14}$. Il a notamment été relevé que cette approche avait le mérite d'assurer une certaine tranquillité d'esprit aux bailleurs, tout en laissant aux locataires suffisamment de temps pour faire valoir leurs droits ${ }^{15}$.

[10] Mais selon la Cour d'appel civile du Tribunal cantonal Vaudois et une autre partie de la doctrine, il faut au contraire considérer que chaque versement effectué par le locataire fait partir un délai de dix ans ${ }^{16}$. À l'appui de cette solution, il est relevé, selon nous à raison, que ce n'est qu'au moment où un versement est effectué que le droit au remboursement prend naissance, et devient donc exigible (art. $130 \mathrm{CO})^{17}$. Il paraît en effet difficile d'admettre qu'une créance en remboursement d'un versement puisse naître avant le versement.

13 Voir ATF 140 III 583 consid. 3.3.1; Lachat/Stastny (note 1), p. 498 N 3.6.1 et les références citées.

14 Arrêt de la Cour de justice GE ACJC/1170/2018 du 3 septembre 2018, CdB 4/18 consid. 2.2. Aucun recours n'a apparemment été déposé devant le Tribunal fédéral ; voir à cet égard LAUre MeYer, Contestation du loyer initial, Délai de prescription, in CdB 4/18, p. 128 ss, p. 132. En doctrine, voir David LACHAT, Le bail à loyer, Lausanne 2008, p. 398 N 2.4.7 ( "mais au plus tard dix ans après la conclusion du bail »). Toutefois, dans la dernière édition de l'ouvrage, LACHAT/Stastny (note 1), p. 498 N 3.6.1, il est précisé « mais au plus tard dix ans après la naissance de celui-ci ». Voir également Cosima Trabichet-Castan/Jacques Johner, Les limites posées à l'admission d'une requête en fixation du loyer initial, Plaidoyer 3/19, p. 32 ss, p. 35 ; Meyer (note 14), p. 132.

15 Trabichet-Castan/Johner (note 14), p. 35.

16 Arrêt de la Cour d'appel civile VD HC/2019/723 du 8 juillet 2019 consid. 3.3. Cet arrêt se réfère à DAviD SchwANinger, in Jürg Müller (édit.), HAP Wohn- und Geschäftsraummiete, Beraten und Prozessieren im Immobiliarmietrecht, Bâle 2016, p. 423 N 10.20, qui traite des transactions couplées. Voir également FETTER (note 2), p. 267 N 582. Apparemment aussi dans ce sens François Bohnet, Les termes et délais en droit du bail à loyer, in $13^{\text {ème }}$ Séminaire sur le droit du bail, Neuchâtel 2004, p. 1 ss, p. 17, qui précise que la créance en restitution se prescrit au plus tard « dix ans après l'enrichissement du bailleur ».

17 Arrêt de la Cour d'appel civile VD HC/2019/723 du 8 juillet 2019 consid. 3.3 ; Fetter (note 2), p. 267 N 582. Voir aussi Alfred Koller, OR AT, Schweizerisches Obligationenrecht Allgemeiner Teil, 4 ème éd., Berne 2017, p. 612 N 33.12, qui s'exprime de manière générale sur le délai de dix ans de l'art. 67 al. 1 CO et se réfère à l'ATF 64 II 132 consid. 2, où il est précisé que « l'action qui dérive de la prestation faite sans cause, la condictio indebiti notamment, naît au moment où la prestation est fournie »; FrÉDÉRIC KrausKopf, in Peter Gauch/Viktor Aepli/Hubert Stöckli (édit.), Präjudizienbuch OR, 9ème éd., Zurich/Bâle/Genève 2016, art. 67 N 4 . Voir aussi l'ATF 114 II 131 consid. 3 (arrêt «Picasso »), où le Tribunal fédéral relève que si un acheteur invoque un vice du consentement, la naissance du droit au remboursement, et par conséquent le délai de prescription de dix ans de l'art. 67 al. 1 $\mathrm{CO}$, correspond au moment du versement ( « mit dem Zeitpunkt der Leistung »). S'agissant des créances de nature contractuelle en remboursement des loyers, le Tribunal fédéral estime que chaque versement fait partir un délai. Voir ATF 130 III 504 consid. 8. Dans ce sens également, mais pas spécialement au sujet du remboursement de loyers, voir ATF 143 III 348 consid. 5.3. 


\section{Les paiements volontaires au sens de l'art. 63 al. 1 CO}

[11] En vertu de l'art. 63 al. 1 CO, « celui qui a payé volontairement ce qu'il ne devait pas ne peut le répéter s'il ne prouve qu'il a payé en croyant, par erreur, qu'il devait ce qu'il a payé ». En d'autres termes, le locataire ne peut pas obtenir le remboursement de versements qu'il a effectués volontairement et sans erreur ${ }^{18}$.

[12] A-t-on affaire à des paiements volontaires? Vu que le loyer est nul dès la conclusion du contrat, le locataire ne semble pas avoir d'obligation de payer le loyer tant que celui-ci n'a pas été fixé judiciairement. Le Tribunal fédéral semble en tout cas être de cet avis puisqu'il soutient qu'en cas de nullité du loyer, le locataire ne peut pas être en demeure tant que le loyer n'a pas été fixé judiciairement ${ }^{19}$. Les paiements effectués par un locataire alors que le loyer est nul doivent donc vraisemblablement être considérés comme des paiements volontaires ${ }^{20}$. Il en irait différemment si l'on admettait - selon nous à tort - que le locataire a l'obligation de continuer à payer le loyer, comme cela a parfois été défendu en doctrine ${ }^{21}$.

[13] Mais s'agit-il de versements effectués sans erreur? Selon le Tribunal fédéral, le locataire n'est pas censé savoir que l'absence d'utilisation de la formule officielle entraîne la nullité du loyer ${ }^{22}$. Autrement dit, l'erreur du locataire est présumée ${ }^{23}$.

[14] Qu'en est-il cependant du locataire qui paie le loyer alors qu'il sait, par exemple parce qu'il a consulté un mandataire professionnel, que l'absence d'utilisation de la formule officielle entraîne la nullité du loyer? Dans ce cas, il faut apparemment considérer que le locataire n'est plus dans $l^{\prime}$ 'erreur ${ }^{24}$. Le locataire peut certes avoir des doutes quant au montant à payer; il est toutefois généralement défendu que celui qui a des doutes n'est pas dans l'erreur ${ }^{25}$.

[15] À partir du moment où il sait que l'absence d'utilisation de la formule officielle entraîne la nullité du loyer, le locataire se trouve dans une position inconfortable. S'il paie l'entier du loyer convenu, il risque de ne pas pouvoir obtenir le remboursement du trop-versé, car les versements pourraient être jugés volontaires et sans erreur. Mais il parait également risqué pour le locataire

18 Vu que le bailleur ne refuse pas la prestation, on ne se trouve pas dans le cas exceptionnel où un créancier peut obtenir le remboursement d'un paiement effectué volontairement et sans erreur. Sur cette exception, voir Gauch/Schluep/Schmid (note 7), N 1576 ss.

19 ATF 137 III 547 consid. 2.3 ; ATF 120 II 341 consid. 6c ; Dietschy-Martenet (note 1), art. 270 N 83.

20 Dans ce sens Thomas Koller, Einmal mehr : Fragwürdiges im Mietrecht vom Mon-Repos, in : Jusletter 20 novembre 2017, Rz 8 .

21 Voir Hans Bätтıg, in Jürg Müller (édit.), HAP Wohn- und Geschäftsraummiete, Beraten und Prozessieren im Immobiliarmietrecht, Bâle 2016, p. 59 N 1.131. D’un avis contraire : Koller (note 20), Rz 8, qui estime que la position de Hans Bättig est «fragwürdig ». La situation est différente si le locataire conteste un loyer initial valablement notifié (art. 270 al. 1 CO). Dans ce cas, le bail reste en vigueur durant la procédure, sous réserve de mesures provisionnelles (art. 270e CO), et le locataire doit continuer à payer son loyer. Dans ce sens Fetter (note 2), p. $113 \mathrm{~N} 246$.

23 ATF 113 II 187 consid. 1a ; arrêt du TF 4A_254/2016 du 10 juillet 2017 consid. 3.1.3.1 ; Fetter (note 2), p. 269 N 586.

24 Dans ce sens Carron (note 12), p. 38. Voir aussi Koller (note 20),Rz 8 ; Alfred Koller, Die Kondiktionssperre von Art. 63 Abs. 1 OR, PJA 2006, p. 468 ss, p. 470, qui se réfère au locataire qui sait qu'une augmentation de loyer est nulle mais s'acquitte tout de même du loyer afin d'éviter un conflit avec le bailleur. Les conditions permettant d'admettre que le locataire n'est plus dans l'erreur semblent donc correspondre aux conditions qui permettent d'admettre que le délai de prescription relatif de trois ans de l'art. 67 al. 1 CO commence à courir. Dans ce sens Carron (note 12), p. 38.

25 Gauch/Schluep/Schmid (note 7), p. 387 N 1535 ; Schulin (note 7), art. 63 N 4 ; Koller (note 17), p. 564 N 31.30. D'un avis plus nuancé : Chappuis (note 7), art. $63 \mathrm{~N} 9$. 
de cesser de payer le loyer ${ }^{26}$. Le Tribunal fédéral a certes admis qu'en cas de nullité du loyer le locataire ne peut pas être en demeure tant que le loyer n'a pas été fixé judiciairement. L'invocation de la nullité pourrait toutefois être jugée abusive (art. 2 CC) $)^{27}$.

[16] Dans une telle situation, il semble que le locataire a intérêt à payer le loyer, mais en faisant des réserves ${ }^{28}$. Si le locataire fait des réserves, on ne peut en effet pas lui opposer l'art. 63 al. 1 CO lorsqu'il demande par la suite le remboursement du trop-versé, car son comportement n'est pas contradictoire $^{29}$, et il ne risque pas de tomber en demeure.

[17] À noter que si le loyer a été fixé provisoirement dans le cadre de mesures provisionnelles, c'est bien sûr ce loyer que doit payer le locataire ${ }^{30}$.

\section{L'augmentation du loyer après la conclusion du contrat}

[18] Une hausse de loyer en cours de bail valablement notifiée au moyen d'une formule officielle et non contestée par le locataire guérit le vice de forme initial ${ }^{31}$. Si le locataire ne conteste pas une telle hausse, il n'a donc plus de créance en restitution pour les montants versés après l'entrée en vigueur de la hausse ${ }^{32}$. Il conserve en revanche une créance en restitution du trop-versé pour la période antérieure ${ }^{33}$.

\section{L'intérêt à agir même lorsque la créance est prescrite}

[19] Dans plusieurs arrêts, le Tribunal fédéral a affirmé que " puisque le législateur n’a pas prévu de règle limitant l'invocation du vice de forme dans le temps, par exemple à la durée du bail, seules les règles de la prescription de l'action en enrichissement illégitime peuvent constituer une limite à l'intérêt du locataire à agir en fixation judiciaire du loyer $»^{34}$.

[20] Il s'agit d'une formulation malheureuse, qui pourrait laisser croire que le locataire ne peut jamais avoir intérêt à agir en fixation du loyer initial si la créance en remboursement du trop-versé est prescrite. En réalité, comme cela a déjà été relevé par plusieurs auteurs, si le bail est toujours

26 Dans ce sens Koller (note 20), p. 3 N 8.

Voir ATF 137 III 547 consid. 2.3 ; Koller (note 20), Rz 8; Dietschy-Martenet (note 1), art. 270 N 83.

Dans ce sens Koller (note 20), Rz 8. Voir aussi Lachat/Stastny (note 1), p. 512 N 4.6.6, qui évoquent le cas d'une hausse de loyer nulle pour vice de forme.

29 Gauch/Schluep/Schmid (note 7), p. 387 N 1535 ; Koller (note 17), p. 569 N 31.41.

30 Sur les mesures provisionnelles, voir Dietschy-Martenet (note 1), art. $270 \mathrm{~N} 83$ et les références citées.

31 Arrêt du TF 4A 256/2015 du 17 septembre 2015 consid. 3.4 ; Blaise Carron, Droit du Bail 2016 p. 33 ss, p. 35 ; Fetter (note 2), p. 123 N 266 s. ; Lachat/Stastny (note 1), p. 495 N 3.5.3. D'un avis contraire : Beat Rohrer, in :

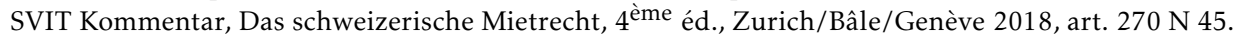

32 Arrêt du TF 4A 256/2015 du 17 septembre 2015 consid. 3.4 ; CArron (note 31), p. 35 ; Fetter (note 2), p. 123 N 266 s. Lachat/Stastny (note 1), p. 495 N 3.5.3 ; Peter Higi, in : Peter Gauch/Jörg Schmid (édit.), Zürcher Kom-

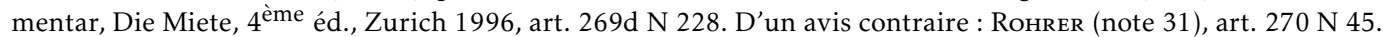

33 Arrêt du TF 4A 256/2015 du 17 septembre 2015 consid. 3.4 ; CArron (note 31), p. 35 ; Fetter (note 2 ), p. 123 N 266 s. ; Higi (note 32), art. 269d N 228 ; Lachat/Stastny (note 1), p. 495 N 3.5.3. D'un avis contraire : Rohrer (note 31 ), art. $270 \mathrm{~N} 45$.

34 ATF 140 III 583 consid. 3.2.3; arrêt du TF 4A_254/2016 du 10 juillet 2017 consid. 3.1.3.1 ; arrêt du TF 4A_517/2014 du 2 février 2015 consid. 4.1.2. 
en vigueur, le locataire conserve un intérêt à agir en fixation du loyer initial afin de réduire les loyers futurs ${ }^{35}$.

\section{Le droit transitoire en matière de prescription}

[21] Une révision importante du droit de la prescription est entrée en vigueur le $1^{\mathrm{er}}$ janvier $2020^{36}$. S'agissant de l'art. 67 al. 1 CO, la seule modification concerne la durée du délai relatif, qui est passée d'une année à trois ans.

[22] Selon la nouvelle version de l'art. 49 al. 1 du Titre final du CC, aussi entrée en vigueur le $1^{\text {er }}$ janvier 2020, «lorsque le nouveau droit prévoit des délais de prescription plus longs que l'ancien droit, le nouveau droit s'applique dès lors que la prescription n'est pas échue en vertu de l'ancien droit».

[23] Par conséquent, si le délai relatif de l'art. 67 al. $1 \mathrm{CO}$ a commencé à courir sous l'ancien droit et n'est pas échu au moment de l'entrée en vigueur du nouveau droit, le délai du nouveau droit (trois ans) s'applique.

\section{Conclusion}

[24] La prescription de la créance du locataire en remboursement du trop-versé en cas de nullité du loyer initial pose des questions délicates. Il faut selon nous notamment retenir que chaque versement effectué par le locataire fait partir un délai de prescription de dix ans. Il faut également admettre qu'à partir du moment où le locataire sait que le loyer initial est nul, il doit émettre des réserves lorsqu'il paie le loyer s'il ne veut pas se voir opposer l'art. 63 CO. Enfin, le locataire peut avoir un intérêt à agir en fixation du loyer initial même si sa créance en remboursement est prescrite.

Laurent Bieri, Professeur à l’Université de Lausanne.

Semsija Etemi, assistante-diplômée et doctorante à l’Université de Lausanne.

Les auteurs remercient Mme Joséphine De Montpellier, assistante-diplômée et doctorante à l'Université de Lausanne, pour sa relecture attentive du manuscrit et pour ses commentaires.

35 Voir Carron (note 12), p. 37 ; Dietschy-Martenet (note 1), art. 270 N 81a.

36 Sur le nouveau droit de la prescription, voir François Bohnet/Anne-Sylvie Dupont (édit.), Le nouveau droit de la prescription, Bâle 2019 et Pascal Pichonnaz/Franz Werro (édit.), Colloque du droit de la responsabilité civile 2019, Le nouveau droit de la prescription, Berne 2019. 